\title{
Techniques of FECG signal analysis: detection and processing for fetal monitoring
}

\author{
M. A. Hasan, M. I. Ibrahimy \& M. B. I. Reaz \\ Department of Electrical and Computer Engineering, \\ International Islamic University Malaysia, Kuala Lumpur, Malaysia
}

\begin{abstract}
Fetal heart rate monitoring is a technique for obtaining important information about the condition of a fetus during pregnancy and labor, by detecting the FECG signal generated by the heart of the fetus. The ultimate reason for the interest in FECG signal analysis is in clinical diagnosis and biomedical applications. The extraction and detection of the FECG signal from composite abdominal signals with powerful and advance methodologies is becoming a very important requirement in fetal monitoring. The purpose of this review paper is to illustrate the various methodologies and algorithms on FECG signal detection and analysis to provide efficient and effective ways of understanding the FECG signal and its nature. A comparative study has been carried out to show the performance of various methods. This paper opens up a passage to biomedical researchers, physicians and end users to advocate an excellent understanding of FECG signal and its analysis procedures for fetal heart rate monitoring system by providing valuable information to help them in developing more dominant, flexible and resourceful applications.
\end{abstract}

Keywords: abdominal ECG, MECG, FECG, fetal heart rate, QRS complex.

\section{Introduction}

Fetal heart rate (FHR) variations during pregnancy and labor have commonly been observed as indirect indications of fetal conditions. An abnormal fetal heart rate or pattern may mean that the fetus is not getting enough oxygen or there are other problems. As a result FHR carries a significient imporatnce of clinical prespectives. 
During the last decades, FHR monitoring has been extensively used for intraand antepartum monitoring to assess fetal well-being. It is commonly used as a screening modulus of the fetus to detect possible fetal problems in advance that could result in irreversible neurological damage or even fetal death during labor. FHR has become a routine physiological measurement both during labor and the antenatal period when certain pregnancy risk factors have been identified. It has been classified that there are two methods of FHR monitoring during labor and delivery: auscultation and electronic fetal monitoring. The choice of the method depends on how a pregnant women's labor is going and her risk of problems.

Electronic fetal monitoring can be external (outside), internal (inside), or both. Internal monitoring involves placement of a small plastic device about the size of a pencil eraser through the cervix. A spiral wire called the fetal scalp electrode is placed just beneath the skin of the fetus's scalp. The fetal scalp electrode then transmits direct information about the fetus's heart rate through a wire to the fetal monitor that prints out the information. Because the internal fetal monitor is attached directly to the fetus, the fetal heart rate signal is sometimes much clearer and more consistent than with an external monitoring device. But, there may be a slight risk of infection with internal monitoring. The scalp electrode may also cause a mark or small cut on the fetus's head, but this usually heals quickly. By definition, external fetal monitoring is done through the skin and is not meant to be invasive. Sensitive electrodes are placed on mother's abdomen over conducting jelly that can sense both FHR, and the strength and duration of uterine contractions. Some FHR monitoring techniques are highlighted in terms of patient's benefits and maintenance.

\section{FHR monitoring techniques}

The analysis of FHR has become a widely accepted means of monitoring fetal status [1]. The most common means of acquiring this data is Doppler ultrasound, with fetal scalp electrodes also often used during labor. The fetal electrocardiogram (FECG) can also be acquired using surface electrodes on the mother's abdomen, whilst the fetal magneto-cardiogram (FMCG) is detectable using SQUID magnetometers [2] and fetal phonocardiography (FPCG) also allows the heart sounds to be detected and hence FHR calculated [3]. The majority of FHR analysis is performed using a bedside monitor over a relatively short period, with the mother to be in a recumbent position. Obviously, a fetal scalp electrode cannot be used for antepartum; the instrumentation required for the acquisition of the FMCG is too cumbersome for ambulatory use; whilst FPCG is felt to be too susceptible to movement artifacts. This left Doppler ultrasound and the abdominal ECG (AECG) as the viable options.

The advantage of the Doppler ultrasound technique is that it can be virtually assured that a recording of FHR will be obtained. The disadvantages of such systems require intermittent repositioning of the transducer and they are only suitable for use with highly trained midwifes. The major limitation of the Doppler ultrasound technique is its sensitivity to movement. The movement of the patient can result in Doppler-shifted reflected waves, which are stronger than 
the cardiac signal. In addition, the effect of ultrasound on the fetus and young infant is not completely clear hence ultrasound is not recommended for long term monitoring [4]. In contrast, methods utilizing the AECG signal have a better prospect for long-term monitoring using signal processing techniques [5]. The abdominal ECG signal can also be used for antepartum non-invasive FHR determination through the detection of small fetal cardiac potentials at the surface of the maternal abdomen [6]. Its advantage is that it is totally noninvasive and unobtrusive, has relatively low power requirements and can be used over extended (e.g. 24 hours) periods. The major disadvantage is that AECG often has a very low signal to noise ratio due to the interference mainly maternal electrocardiogram (MECG), electromyogram (EMG), and motion artifact. Thus, the acquisition of the FECG from the AECG cannot be guaranteed. To overcome the above problems, some multiple-lead algorithms use the thoracic MECG to cancel the abdominal MECG [7], though this is inconvenient for the patient during long-term monitoring. Hence, to make the AECG suitable for the detection of the FECG, the signal to noise ratio (SNR) must be enhanced and then much signal processing is needed.

This paper firstly gives a brief clarification about FECG signal. This is followed by highlighting the up to date detection, extraction and classification methods of FECG signal along with a comparison study.

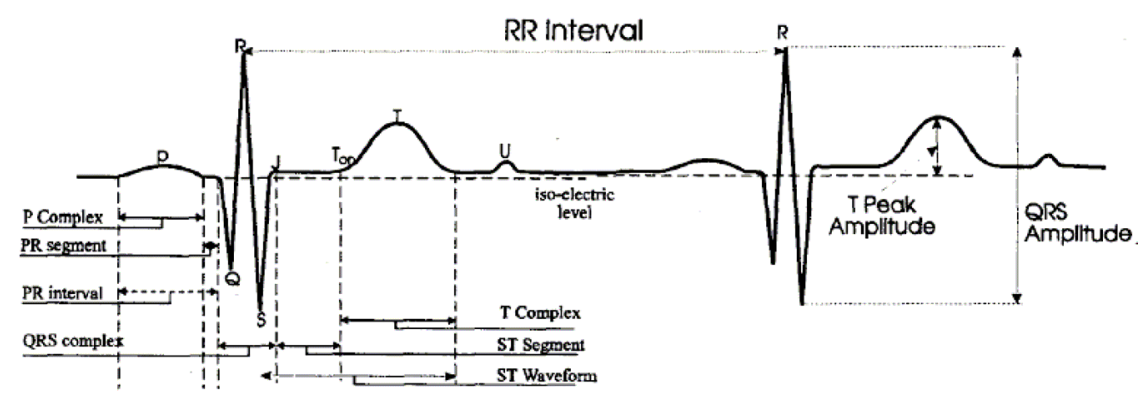

Figure 1: Fetal ECG is showing key features: the QRS complex.

\section{Clinical importance of FECG morphology}

Biomedical signal means a collective electrical signal acquired from any organ that represents a physical variable of interest. FECG is a biomedical signal that gives electrical representation of FHR. Sometimes the FECG is the only information source in early stage diagnostic of fetal health. The signal as shown in Figure 1 is an electric signal produced by the contraction of the heart's muscle called myocardium. It is composed of three parts: i) the P-wave reflects the contraction of the atriales, ii) the QRS-complex is associated with the contraction of the ventricles, due to the magnitude of the R-wave, it is extremely reliable, 
iii) the T-wave, which corresponds to the repolarisation phase and follows each heart contraction. The delay associated to the R-R interval leads to the heartbeats frequency. The R-R interval gives useful information about the heart condition.

Morphologies of interest include the shape, size, and duration of individual and groups of FECG waveforms as well as the various ratios relating these quantities to each other. The FECG displays the muscle particular shapes that can serve as direct facts of specific malfunctions and abnormalities.

\section{Interfaces and noises affecting the FECG signal}

The FECG signal is interfered by various noises with unknown spectral and temporal characteristics. The main source of interference is the maternal electrical activity, the amplitude of which is much higher than the amplitude of the fetus electrical activity, which is often completely masked by the former. The FECG signals are often obscured by electrical noise from other sources. Common ECG noise sources, such as power line interference, muscle contractions, respiration, skin resistance interference, instrumental noise, in addition to electromyogram and electrohysterogram due to uterine contractions, can corrupt FECG signals significantly [8]. The signal processing algorithm needs to remove the maternal ECG complexes, reduce the effects of motion artifact and muscle noise, and then enhance the fetal QRS complexes before they can be consistently detected.

\section{FECG signal detection}

Several methods have been proposed for detecting the FECG signal from abdomen ECG signal. In 1990, Tal and Akselrod [9] proposed a discrete Fourier transform method for the detection of FHR from AECG recordings. The transform method enables the detection of FHR from AECG signals in which the fetal signal is barely visible. Following the elimination of the MECG contribution to the signal, they computed a triple parametric transform function by multiplying the signal by their analyzing functions and integrating the result. In general the method can be applied to handle weak, quasi periodic, sharp signals of various origins. Stoughton et al. [10] in 1990 had a theory saying that adaptive least mean square (LMS) linear prediction methods can be used for fetal heart tone signature analysis and detection in the presence of background acoustic noise. Adaptive signal processing methods are presented in support of a non-invasive ambulatory FHR monitor. Subsequent evaluation of the detected fetal heart tone events are used to determine the instantaneous heart rate. Preliminary investigation has indicated that linear prediction is feasible for detecting the fetal heart tones in an advanced acoustic fetal heart rate monitor.

Kam and Cohen [11] proposed two architectures for the detection of fetal ECG. The first is a combination of an IIR adaptive filter and Genetic algorithm (GA), where the GA is recruited whenever the adaptive filter is suspected of reaching local minima. The second is an independent GA search without the adaptive filter. The main disadvantage of an IIR filter is that the error surface is 
not quadratic but a multi modal surface. So, the presence of the GA forces the algorithm to overcome the local minima and reach the global solution. The quality of the extracted FECG using this IIR-GA adaptive filter is superior to that obtained using the GA alone.

In 2000, Lai and Shynk [12] proposed an adaptive algorithm for detecting and separating fetal and maternal heart beats from data containing both fetal and maternal QRS complexes. This algorithm has a relatively low computational complexity, and does not require reference signals to cancel the maternal QRS complexes and classifies the combined heart-rate data as a series of fetal, maternal, and noise events using a technique of template matching. Peters et al. [13] in 2006 developed an algorithm that calculates the heart rate based on cross correlation. By using the developed algorithm for calculating the FHR, multielectrode electrical measurements on the maternal abdomen now can be used for fetal monitoring in relatively early stages of pregnancy or other situations where ECG amplitudes are low or noise levels are high. In order to improve the detection accuracy, efficient signal processing is needed. Also FECG extraction problem is not easily solved by conventional filtering techniques. Linear filtering in the Fourier domain fails since the spectral content of all the three components, MECG, FECG and noise are rather similar and overlap. Some problem descriptions can be discussed followed by the past researches that have been done in the area of FECG extraction.

\subsection{Wavelet transform}

The Wavelet transform (WT) is an efficient mathematical tool for local analysis of non-stationary and fast transient signals. One of the main properties of WT is that it can be implemented by means of a discrete time filter bank. In 1996, Echeverria et al. [14] developed a procedure, wavelet analysis and pattern matching (WA-PM) for the off-line processing of AECG, on which they assumed that this signal, $s(t)$, can be mathematically described by the equation $s(t)=r(t) \cdot[f(t)+m(t)+n(t)]$. The terms $f(t), m(t)$ and $n(t)$ refer respectively to fetal, maternal, and Gaussian noise components, all of them affected by a modulation factor $r(t)$ that cause baseline wandering [15]. The procedure involves two-stages: the first consists in a preprocessing stage for the suppression of low and high frequency additive noise based on an optimal wavelet multiresolution decomposition, and the second cancels the maternal QRS complexes by means of pattern matching and template subtraction. The fetal QRS complexes can be easily identified from the resulting signal by applying a QRS detection algorithm. Again in 1996, Papadimitriou et al. [16] used Wavelet transform to denoise FHR signals. His WT approach effectively removes transient spikes and reduces noise (both Gaussian and colored) without destroying the high frequency information content of the signal (as the traditional low pass filtering does). Mochimaru et al. [17] (2002) used wavelet based methods to detect the FECG. To remove the large baseline fluctuations in the signal as well as to remove the noise, multiresolution analysis (MRA) was used. 
In 2004, Karvounis et al. [18] discovered that the complex continuous wavelet transform (CCWT) and modulus maxima theory can also be used to detect of the QRS complexes of the fetus cardiac activity using multi-channel maternal ECG recordings. For a non-stationary signal, CWT can be used to identify stationary sections of the data stream and to locate and characterize singularities. This approach improves the signal to noise ratio, which allows reliable FHR variation to be estimated under very weak signal environment.

\subsection{Time-frequency approach}

In 2006, Karvounis et al. [19] showed three-stage method for FHR extraction based on time-frequency analysis, from AECG recordings. The method is based on the analysis of leads of the AECG signal. In the first stage the maternal Rpeaks and fiducial points (QRS onset and offset) are detected, using timefrequency analysis, and the maternal QRS complexes are eliminated. The second stage locates the positions of the candidate fetal R-peaks, using complex wavelets and pattern matching theory techniques. In the third stage, a histogrambased technique is used as the fetal R-peaks that overlap with the maternal QRS complexes are found.

\subsection{Artificial intelligence}

Artificial Intelligence (AI) techniques mainly based on Neural network (NN) have been proposed for processing FECG signal. Neural network is a computing technique that evolved from mathematical models of neurons and systems of neurons. During recent years, NN has become a useful tool for categorization of multivariate data. This kind of technique is very useful for real-time application like FECG signal recording and analysis. In 1994, Liszka-Hackzell [20] showed that the categorization process for the FHR patterns, in which NN was used, can be reliable and well with the manual categorization. Since the categorization by NNs is very fast and does not involve human efforts, it may be useful in patient monitoring. It used only two types of networks: the back-propagation network (BP) and the self-organizing map network (SOM).

In 1999, Magenes et al. [21] applied Neural and Fuzzy classifiers to detect accurately normal and pathological fetal states. They showed the Neural and Fuzzy classifiers can improve the diagnostic information contained in CTG signals. Selvan and Srinivasan [22] in 2000 had a theory saying that the two popular adaptive filtering techniques, namely adaptive noise cancellation and adaptive signal enhancement, is an efficient technique for processing of abdominal FECG by using NN. The proposed technique performs better than the noise canceller alone or a cascade connection of both noise canceller and signal enhancer.

Fetal ECG extraction using adaptive liner neural network was proposed by Reaz and Wei [23] in 2004. The Adaptive Linear Neural Network Filter is trained to cancel out the maternal signal. According to their proposed method, it is considered that the network error equals abdominal ECG minus maternal ECG, which is the fetal ECG. This method is better than conventional filtering 
because subtraction is used instead. It can avoid eliminating desirable signal. In 2005, Warrick et al. [24] used the combined tools of signal processing and Neural networks to develop an automated technique to detect the FHR patterns of baseline, acceleration, and deceleration.

\subsection{ICA and blind source separation}

Some others Artificial Intelligence techniques mainly based on Blind Source Separation (BSS) proposed for processing FECG signal. During recent years, BSS and Independent Component Analysis (ICA) has become a positive tool for categorization of multivariate data.

In 2000, de Lathauwer et al. [25] proposed the emerging technique of ICA, also known as BSS, as an innovating way to solve a classical problem in biomedical engineering, namely the extraction of the FECG from multilead potential recordings on the mother's skin. From a conceptual point of view, ICA is a very ambitious approach; it aims at the direct reconstruction of the different statistically independent bioelectric source signals, as well as the characteristics of their propagation to the electrodes. It is nonparametric and is not based on pattern averaging, which could hamper the detection and analysis of atypical fetal heartbeats.

Barros and Cichocki [26] discovered a semi-blind source separation algorithm to solve the fetal ECG extraction problem in 2001. This algorithm requires a priori information about the autocorrelation function of the primary sources, to extract the desired signal (FECG). They do not assume the sources to be statistically independent but they assume that the sources have a temporal structure and have different autocorrelation functions. The main problem with this method is that if there is fetal heart rate variability, as is the case when the fetus is not healthy, the priori estimate of the autocorrelation function of the fetal ECG may not be appropriate.

By using ICA method, in 2004, Burghoff and Van Leeuwen [27] have given their theory about the separation of fetal and maternal magnetocardiographic signals in twin pregnancy. ICA, which uses higher order statistics to decompose the signal into statistical independent components, has already been used in single pregnancies to distinguish between maternal and fetal ECG signals [28]. The results showed that the maternal and fetal components can be separated from each other as well as from other sources of noise and artifacts.

In 2004, Chareonsak et al. [29] proposed a real time BSS method that can be used to separate the FECG from the AECG effectively.

Recently, Najafabadi et al. [30] also applied the ICA for the separation of FECG and MECG signal from the AECG in 2005. It is concluded that ICA works magnificent in order to extract FECG even in SNR $=-200 \mathrm{~dB}$ using simulated data without quantification noise. It showed that the performance was drastically decreased in existence of quantification noise.

There are some other models proposed by various researchers for the purpose of FECG signal processing. The sketch out of the foremost methods is given in Table 1. 
Table 1: $\quad$ Sketch out of the foremost methods.

\begin{tabular}{|c|c|}
\hline Method & Advantage/Disadvantage \\
\hline $\begin{array}{l}\text { Fourier } \\
\text { Transform }\end{array}$ & $\begin{array}{l}\text { - When the FECG is obscured by noise and the peak } \\
\text { detection algorithm fails, a transform method might still } \\
\text { detect the FHR proficiently. } \\
\text { - SNR is an averagely high. } \\
\text { - In the case of weak signals having small duty cycle, this } \\
\text { tool might sometimes fail to detect the average periodicity } \\
\text { because of small correlation between the signals. }\end{array}$ \\
\hline $\begin{array}{l}\text { Least Mean } \\
\text { Square }\end{array}$ & $\begin{array}{l}\text { - Feasible for fetal heart tone signature identification and } \\
\text { analysis in the presence of background acoustic noise. }\end{array}$ \\
\hline $\begin{array}{l}\text { Complex } \\
\text { Continuous } \\
\text { Wavelet } \\
\text { Transform }\end{array}$ & $\begin{array}{l}\text { - Performs well and the accuracy of the method is high. } \\
\text { - Algorithm's parameters increase the system's efficacy. } \\
\text { - Computationally fast and excels in performance } \\
\text { - Able to extract the maternal signal which can be useful } \\
\text { for parallel monitoring of the mother's health. }\end{array}$ \\
\hline $\begin{array}{l}\text { Wavelet } \\
\text { Transform }\end{array}$ & $\begin{array}{l}\text { - Coherent average can get more accurate reference. } \\
\text { - Can be obtained to smooth the baseline drift. } \\
\text { - } \text { Require only one abdominal signal for fetal QRS } \\
\text { extraction and maternal QRS cancellation. } \\
\text { - More flexible and effective tool for FHR signals } \\
\text { denoising than the traditional filtering techniques. }\end{array}$ \\
\hline $\begin{array}{l}\text { Time- } \\
\text { Frequency } \\
\text { Analysis }\end{array}$ & $\begin{array}{l}\text { - Three leads are used for FECG extraction. } \\
\text { - Spectrum produced by Wigner-Ville distribution (WVD) } \\
\text { which displays very good localization properties } \\
\text { - The main drawback of the method is the difficulty to } \\
\text { extract the fetal R-peaks in noisy background or in cases } \\
\text { where the FECG is not distinguishable. }\end{array}$ \\
\hline $\begin{array}{l}\text { Artificial } \\
\text { Neural } \\
\text { Networks }\end{array}$ & $\begin{array}{l}\text { - Very fast and does not involve human efforts for } \\
\text { categorization. } \\
\text { - Neural networks can offer the computational power of } \\
\text { non-linear techniques. } \\
\text { - Sometimes it does not estimate the exact base line value } \\
\text { and its precision limited by the number of classes. }\end{array}$ \\
\hline $\begin{array}{l}\text { Independent } \\
\text { Component } \\
\text { Analysis and } \\
\text { Blind Source } \\
\text { Separation }\end{array}$ & $\begin{array}{l}\text { - Relatively SNR is high. } \\
\text { - Efficient both in batch and on-line operation modes. } \\
\text { - Fast and efficient approach for the preprocessing of } \\
\text { multiple signals of interest. } \\
\text { - No specific prior knowledge is required in order to } \\
\text { identify the components generated from different sources. } \\
\text { - Often require a large number of recorded leads to reach } \\
\text { reliable FECG extraction. }\end{array}$ \\
\hline
\end{tabular}




\section{Conclusion}

Detail analysis of the FECG during labor could provide valuable additional information about the fetus well-being as well as to assist clinicians in reducing incidents of unnecessary medical intervention without altering current patient handling routines. So the aim of this paper was to provide concise information about FECG and reveal the different methodologies to analyze the signal. Techniques for FECG signal detection and extraction from the composite abdominal signal were discussed along with their advantages and drawbacks. This revision obviously points up the various types of FECG signal analysis techniques so that accurate methods can be applied during any medical diagnosis, biomedical research and end user applications.

\section{Acknowledgement}

The authors would like to express sincere gratitude to the Ministry of Science, Technology and Innovation of Malaysia for providing fund for the research under eScienceFund grant (Project No.01-01-08-SF0029).

\section{References}

[1] Van Geijn, H.P. \& Copray, F.J.A., A Critical Appraisal of Fetal Surveillance, Academic Hospital of the Free University, Amsterdam, Netherlands. 1994.

[2] Crowe, J.A., Herbert, J.M., Huang, X.B., Reed, N., Woolfson, M.S., Rassi, D., Zhuravlev, Y.E. \& Emery, S.J., Sequential recording of the abdominal fetal electrocardiogram and magnetocardiogram. Physiological Measurement, 16(1), pp. 43-47, 1995.

[3] Bassil, H.E. \& Dripps, J.H., Real time processing and analysis of fetal phonocardiographic signals. Clin.Phys.Physio1. Meas., pp. 67-74, 1989.

[4] Goodlin, R.C., History of fetal monitoring. Am J Obstet Gynecol, 133(3), pp. 323-352, 1979.

[5] Kanjilal, P.P., Palit, S. \& Saha, G., Fetal ECG extraction from singlechannel maternal ECG using singular value decomposition. Biomedical Engineering, IEEE Transactions, 44(1), pp. 51-59, 1997.

[6] Solum, T., Ingemarsson, I. \& Nygren, A., The accuracy of abdominal ECG for fetal electronic monitoring. J Perinat Med., 8(3), pp. 142-149, 1980 .

[7] Khamene, A. \& Negahdaripour, S., A new method for the extraction of fetal ECG from the composite abdominal signal. Biomedical Engineering, IEEE Transactions, 47(4), pp. 507-516, 2000.

[8] Zarzoso, V., Nandi, A.K. \& Bacharakis, E., Maternal and foetal ECG separation using blind source separation methods. IMA Journal of Mathematics Applied in Medicine \& Biology, 14(3), pp. 207-225, 1997. 
[9] Tal, Y. \& Akselrod, S., Feral heart rate detection by a special transformation method. Proc. IFEE Comp. Soc. Conf. Computers in Cardiology, pp. 275-278, 1990.

[10] Stoughton, J.W., Weber, G.N. \& Pretlow, R.A., Fetal heart rate estimation via adaptive least mean square linear prediction methods. Southeastcon '90. Proceedings IEEE, New Orleans, LA, USA, 1, pp. 260-264, 1990.

[11] Kam, A. \& Cohen, A., Detection of fetal ECG with IIR adaptive filtering and genetic algorithms. IEEE International Conference on Acoustics, Speech, and Signal Processing (ICASSP'99), 4, pp. 2335-2338, 1999.

[12] K.-C. Lai \& Shynk, J.J., A signal separation algorithm for fetal heart-rate estimation. Signals, Systems and Computers Conference Record of the Thirty-Fourth Asilomar, Pacific Grove, CA, USA, 1, pp. 348 - 351, 2000.

[13] Peters, C., Vullings, R., Bergmans, J., Oei, G. \& Wijn, P., Heart rate detection in low amplitude non-invasive fetal ECG recordings. 28th IEEE EMBS Annual International Conference, New York City, USA, pp. 60926094, 2006.

[14] Echeverria, J.C., Ramirez, N., Pimentel, A.B., Rodriguez, R., Gonzalez, R. \& Medina, V., Fetal QRS extraction based on wavelet analysis and pattern matching. Engineering in Medicine and Biology Society, Bridging Disciplines for Biomedicine. Proceedings of the 18th Annual International Conference of the IEEE, 4, pp. 1656-1657, 1996.

[15] Bergveld, Piet., Kolling, Arjan J. \& Peuscher, Jan. H. J., Real-time fetal ECG recording. Biomedical Engineering, IEEE Transactions, 33(5), pp. $505-509,1986$.

[16] Papadimitriou, S., Gatzounas, D., Papadopoulos, V., Tzigounis, V. \& Bezerianos, A., Fetal heart rate signal denoising by processing the wavelet transform modulus maxima, Engineering in Medicine and Biology Society, Bridging Disciplines for Biomedicine. Proceedings of the $18^{\text {th }}$ Annual International Conference of the IEEE, 3, pp. 1029-1030, 1996.

[17] Mochimaru, F., Fujimoto, Y. \& Ishikawa, Y., Detecting the fetal electrocardiogram by wavelet theory-based methods. Progress in Biomedical Research, pp. 185-193, 2002.

[18] Karvounis, E.C., Papaloukas, C., Fotiadis, D.I. \& Michalis, L.K., Fetal heart rate extraction from composite maternal ECG using complex continuous wavelet transforms. Computers in Cardiology, pp. 737-740, 2004.

[19] Karvounis, E.C., Tsipouras, M.G., Fotiadis, D.I. \& Naka, K.K., A method for fetal heart rate extraction based on time-frequency analysis. 19th IEEE Symposium on Computer-Based Medical Systems (CBMS'06), pp. 347347, 2006.

[20] Liszka-Hackzell, J., Categorization of fetal heart rate patterns using neural networks. Computers in Cardiology, Bethesda, MD, USA, pp. 97-100, 1994.

[21] Magenes, G., Signorini, M.G. \& Arduini, D., Detection of normal and pathological fetal states by means of neural and fuzzy classifiers applied to CTG parameters. Engineering in Medicine and Biology, $21^{\text {st }}$ Annual 
Conf. and the 1999 Annual Fall Meeting of the Biomedical Engineering Soc, BMES/EMBS Conference, Atlanta, GA, USA, 2, pp. 936, 1999.

[22] Selvan, S., \& Srinivasan, R., A novel adaptive filtering technique for the processing of abdominal fetal electrocardiogram using neural network. Adaptive Systems for Signal Processing, Communications, and Control Symposium 2000. (AS-SPCC), IEEE, Louise, Alta., Canada, pp. 289-292, 2000.

[23] Reaz, M.B.I. \& Wei, L.S., Adaptive linear neural network filter for fetal ECG extraction. Intelligent Sensing and Information Processing, Proceedings of International Conference, pp. 321-324, 2004.

[24] Warrick, P., Hamilton, E. \& Macieszczak, M., Neural network based detection of fetal heart rate patterns. Proceedings of International Joint Conference on Neural Networks, Montreal, Canada, 4, pp. 2400-2405, 2005.

[25] de Lathauwer, L., de Moor, B. \& Vandewalle, J., Fetal electrocardiogram extraction by blind source subspaceseparation. Biomedical Engineering, IEEE Transactions, 47(5), pp. 567-572, 2000.

[26] Barros, A.K. \& Cichocki, A., Extraction of specific signals with temporal structure, Neural Computation, MIT Press, 13(9), 2001.

[27] Burghoff, M. \& Van Leeuwen, P., Separation of fetal and maternal magnetocardiographic signals in twin pregnancy using independent component analysis (ICA). Neurology and Clinical neurophysiology, 2004.

[28] de Araujo, D., et al., Fetal magnetocardiographic source separation using the poles of the autocorrelation function. Proceedings of the 4th International Symposium on Independent Component Analysis and Blind Signal Separation, Nara, Japan, pp. 833-836, 2003.

[29] Chareonsak, C., Sana, F., Wei, Y. \& Bing, X., Design of FPGA hardware for a real-time blind source separation of fetal ECG signals. Biomedical Circuits and Systems, 2004 IEEE International Workshop, pp. 13-16, 2004.

[30] Najafabadi, F.S., Zahedi, E. \& Ali, M.A.M., A novel model for abdominal electrocardiography of a pregnant woman. Sensors and the International Conference on new Techniques in Pharmaceutical and Biomedical Research, pp. 64 - 68, 2005. 